\title{
Transport Properties of Ordered and Disordered Doped Metallic Nanotubes
}

\author{
Rekha Singh* \\ Research Scholar, University Department of Physics, B. R. A. Bihar University, Muzaffarpur, India \\ *Corresponding author: drrekhasinghbela09@gmail.com
}

\begin{abstract}
We have studied the transport properties of ordered and disordered doped metallic nanotubes. We have presented these properties in periodic chemically metallic nitrogen doped metallic nanotubes. Transport properties with long range correlation have been studied using the Green's function formalism in the frame work of the tight binding approach with effective parameters. Resonant conduction behaviour and ballistic transport have been demonstrated for particular positions of the dopant. These doping patterns have been shown to preserve the Bloach like transport properties and then to avoid Anderson localisation. The conductance response was almost changed because one of the two conductance channels remains true for both armchair and chiral nanotubes. These result reproduce the electronic properties of periodic nitrogen doped graphene. The present result has been drawn for specific chemical substitution of carbon atoms by nitrogen. These remain qualitatively valid for other local modifications of the nanotube by other chemical species by covalent or noncovalent functionalization. The energy of the quasi bound state depend on the specific local modification. We have shown that both axial and screw periodicities gave rise to such a behaviour and that specific disorder preserve their ballistic transport in doped metallic carbon nanotube.
\end{abstract}

Keywords: Bloch, Covalent, Doping, Green's function, Metallic nanotubes, Quasi bound, Symmetry, Transport.

\section{Introduction}

Lin [1] reported strategies for the production of nitrogen doped nanotubes. The local electronic perturbation of a single nitrogen substitution has been observed occasionally by electron energy loss spectroscopy and scanning tunnel microscopy but macroscopic samples are mainly characterized by a disordered distribution of defects with different atomic structures as confirmed by transport measurements and photoemission spectroscopy. The reported properties are related to the Langrange correlation effects predicted for the electronic properties and the quantum transport [2] of nitrogen doped grapheme when chemical doping affects only one of the two sub lattices of grapheme. Poor control of the growth mechanisms has however curbed the development of devices with adjusted intrinsic properties, even of post synthesis chirality sorting in improving [3]. In our works [4] the onsite effective parameters of the carbon atoms are modified around the defect up to a distance dc; $7.5 \mathrm{~A}$ along the surface of the tube.

\section{Result and Discussion}

We have first considered the effect of nitrogen chemical doping on the armchair $(10,10)$ nanotubes as an arche type of metallic system with high symmetry. Axial and screw periodicity of the dopant position were investigated as well as the effect of disorder. Based on these results on a symmetry analysis, we then extended our findings to chiral nanotubes. For the $(10,10)$ nanotubes $\mathrm{d} 0=2.45 \mathrm{~A}$ and the distance between two nitrogen atoms is always set larger than $7 \mathrm{~d} 0$. In present simulations for the quantum conductance $\mathrm{g}(\mathrm{E}) / \mathrm{g} 0$ as a function of the energy for two supercell periods with $\mathrm{n}$ cell being a multiple of 3 or not, i.e., $n$ cell $=15$ and $n$ cell $=14$ corresponding to defect concentrations of $0.166 \%$ and $0.178 \%$ respectively.

\section{Conclusion}

We have found that transport properties of ordered and disordered nitrogen doped metallic carbon nanotubes with long range correlation were studied numerically with a tight binding model. In periodic defective systems, when axial doping is considered, two classes of electronic transport responses are obtained. One quantum conductance plateau settles down around the defect energy only when the period of the structure is a multiple of the Fermi wavelength, because the Bloach like propagating modes survive. Otherwise a conduction gap is predicted.

\section{References}

[1] Lin, H., Lagoute, J., Chacon, C., Arenal, R., Stéphan, O., Repain, V., Girard, Y., Enouz, S., Bresson, L., Rousset, S. and Loiseau, A. (2008), Combined STM/STS, TEM/EELS investigation of $\mathrm{CN}_{x}-\mathrm{SWNTs}$. Phys. Status Solidi B, 245: 1986-1989.

[2] Aurélien Lherbier, Andrés Rafael Botello-Méndez, and Jean-Christophe Charlier, "Electronic and Transport Properties of Unbalanced Sublattice N-Doping in Graphene," Nano Letters 201313 (4), 1446-1450.

[3] Tu X, Manohar S, Jagota A, Zheng M. DNA sequence motifs for structure-specific recognition and separation of carbon nanotubes. Nature. 2009 Jul 9;460(7252):250-3.

[4] Hafid Khalfoun, Patrick Hermet, Luc Henrard, and Sylvain Latil, "B and $\mathrm{N}$ codoping effect on electronic transport in carbon nanotubes," Physical Review B, vol. 81, May 2010. 